\title{
Das Recht auf Datenübertragbarkeit
}

Der Einzelne hat die Befugnis, grundsätzlich selbst über die Preisgabe und Verwendung seiner persönlichen Daten zu bestimmen. Dieses Recht auf informationelle Selbstbestimmung, das das Bundesverfassungsgericht in seinem Volkszählungsurteil im Jahr $1983^{1}$ aus Art. 2 Abs. 1 GG und Art. 1 Abs. 1 GG abgeleitet hat, gilt als Ausgangspunkt für die weitere Entwicklung des Datenschutzrechts. Heute - in Zeiten von Big Data - scheint es wichtiger denn je, dass das Recht auf informationelle Selbstbestimmung wirksam durchgesetzt werden kann. Bekanntermaßen lassen sich Facebook, Google und Co. in ihrer Vorgehensweise aber nicht oder nur wenig von datenschutzrechtlichen Vorschriften beirren. Ein selbstbestimmter Umgang mit personenbezogenen Daten ist somit nur möglich, wenn die Rechte der Betroffenen weiter gestärkt werden.

Die am 25. Mai 2016 in Kraft getretene DS-GVO eröffnet den Betroffenen die Möglichkeit, auf ein vereinheitlichtes System von Betroffenenrechten zurückzugreifen, um so ihrer Selbstbestimmung in einer zunehmend digitalisierten Welt Geltung zu verschaffen. Die DS-GVO normiert unter anderem Löschungs-, Berichtigungs- und Informationsrechte zum Schutz natürlicher Personen bei der Verarbeitung personenbezogener Daten. Diese Rechte sind dem Datenschutzrecht auch schon bisher nicht fremd, das BDSG regelt annährend gleichlautende Ansprüche der Betroffenen.

Der europäische Gesetzgeber beließ es jedoch nicht bei diesen allgemein anerkannten sowie bewährten Rechten. In Art. 20 DSGVO wurden die Betroffenenrechte um ein Recht auf Datenübertragbarkeit ergänzt. Danach hat die betroffene Person das Recht, dass die sie betreffenden personenbezogenen Daten von einem Verantwortlichen, dem die Daten z.B. im Rahmen eines Vertrages ursprünglich bereitgestellt wurden, auf einen anderen Verantwortlichen übermittelt werden. Diese Übermittlung erfolgt entweder direkt zwischen den beiden Verantwortlichen oder über den Betroffenen, indem ihm eine Kopie der Daten übermittelt wird, die in einem zweiten Schritt dem anderen Verantwortlichen - als „Erwerber der Daten“ - zur Verfügung gestellt wird.

Die Normadressaten - insbesondere Anbieter von OnlineDiensten - werden sich in Zukunft mit einigen technischen und rechtlichen Herausforderungen in Bezug auf das Recht auf Datenübertragbarkeit konfrontiert sehen.

Praxisrelevant ist beispielsweise die Problematik der technischen Umsetzbarkeit einer universellen Datenportabilität sowie der Interoperabilität der Daten. Art. 20 Abs. 1 DS-GVO setzt voraus, dass personenbezogene Daten in einem strukturierten, gängigen und maschinenlesbaren Format zu übermitteln sind. Ferner erwähnt Erwägungsgrund 68 der DS-GVO das Erfordernis interoperabler Daten. Dass Unternehmen unterschiedliche Datenformate verwenden, die auf die individuellen Datenverarbeitungssysteme zugeschnitten sind und Kunden binden sollen („Apple-Ökosystem“), könnte auf technischer Ebene zu erheblichen Schwierigkeiten führen. Inwieweit die Übermittlung

\footnotetext{
1 BVerfGE 65, 1
}

einer Datensammlung zwischen zwei Verantwortlichen in $\mathrm{Zu}$ kunft tatsächlich ohne größere Behinderungen umgesetzt werden kann, bleibt abzuwarten. Im Hinblick auf die drakonisch anmutenden Sanktionen bei Verstößen gegen Betroffenenrechte (vgl. Art. 83 Abs. 5 lit. b DS-GVO) sollten sich Internetdienste-Anbieter gleichwohl bemühen, „verkehrsfähige“ Datenformate zu entwickeln.

Ferner ist die Normierung eines Rechts auf Datenübertragbarkeit aus juristischer Perspektive diskussionswürdig. Insbesondere stellt sich die Frage, inwieweit das Recht auf informationelle Selbstbestimmung der Möglichkeit einer Datenportabilität bedarf. Bereits die datenschutzrechtliche Natur der Vorschrift, die anlässlich der systematischen Einordnung in die Betroffenenrechte der DS-GVO erwartet werden könnte, ist zweifelhaft, denn ihren Ursprung findet die Vorschrift vor allem in wettbewerbsrechtlichen Erwägungen. ${ }^{2}$ Ziel der Regelung ist primär die Vermeidung des sog. „Lock-in-Effekts“ sowie die Stärkung des Wettbewerbs auf digitalen Märkten durch einen vereinfachten Anbieterwechsel. ${ }^{3}$ „Lock-in-Effekte“ liegen vor, wenn Nutzer aufgrund von Entscheidungen, die sie in der Vergangenheit getroffen haben, nicht zu einem neuen Netzwerk wechseln, wobei insbesondere Wechselkosten eine Rolle spielen. ${ }^{4}$ Vor diesem Hintergrund lässt sich das Recht auf Datenübertragbarkeit - als „Instrumentarium des Datenschutzrechts" - nur schwerlich in das System der Betroffenenrechte einordnen. Die klassischen Betroffenenrechte wie bspw. Anspruch auf Löschung, Berichtigung, Widerspruch und Auskunft zielen in erster Linie darauf ab, rechtswidrige Datenverarbeitungen zu verhindern und zu unterbinden. Insofern sind Betroffenenrechte als - zumeist reaktive - Abwehrrech$\mathrm{te}^{5} \mathrm{zu}$ klassifizieren. Obwohl die Ausübung des Rechts auf Datenübertragbarkeit zur Folge haben könnte, dass Nutzer über die sie betreffenden Daten eine bessere Kontrolle ausüben ${ }^{6}$ oder lediglich Verträge mit datenschutzfreundlichen Unternehmen abgeschlossen werden und insofern eine indirekte Förderung des Datenschutzes stattfindet ${ }^{7}$, kann nicht von einer - primär - dem Schutz von personenbezogenen Daten dienenden Vorschrift gesprochen werden. ${ }^{8}$

Allerdings erscheint eine eindeutige Zuordnung zum Wettbewerbs- oder Datenschutzrecht auch nicht notwendig. Vielmehr kann Art. 20 DS-GVO als Schnittstelle zwischen Wettbewerbsund Datenschutzrecht verstanden werden. ${ }^{9}$ Sicher ist: Das Recht auf Datenübertragbarkeit fördert die Etablierung personenbezogener Daten als kommerzialisierbares Gut auf dem europäischen „Binnen(daten)markt“.

\footnotetext{
2 Herbst in Kühling/Buchner, DS-GVO, Art. 20 Rn. 4.

3 Jülicher/Röttgen/v. Schönfeld, ZD 2016, 358 (360).

4 Auer-Reinsdorff/Conrad, Handbuch IT- und Datenschutzrecht, §39 Rn. 444.

5 Vgl. Dix in Simitis, BDSG, § 35 Rn. 2.

6 EG 68.

7 Vgl. Kamlah in Plath, BDSG/DSGVO, Art. 20 Rn. 1.

8 Herbst in Kühling/Buchner, DS-GVO, Art. 20 Rn. 4.

9 Vgl. Kamlah in Plath, BDSG/DSGVO, Art. 20 Rn. 2; Jülicher/Röttgen/v. Schön-
} feld, ZD 2016, 358 (360). 